\title{
Biocontrol properties from phyllospheric bacteria isolated from Solanum lycopersicum and Lactuca sativa and genome mining of antimicrobial gene clusters
}

\author{
Claudia Y. Muñoz, Lu Zhou, Yunhai Yi and Oscar P. Kuipers ${ }^{*}$
}

\begin{abstract}
Background: Biocontrol agents are sustainable eco-friendly alternatives for chemical pesticides that cause adverse effects in the environment and toxicity in animals including humans. An improved understanding of the phyllosphere microbiology is of vital importance for biocontrol development. Most studies have been directed towards beneficial plant-microbe interactions and ignore the pathogens that might affect humans when consuming vegetables. In this study we extended this perspective and investigated potential biocontrol strains isolated from tomato and lettuce phyllosphere that can promote plant growth and potentially antagonize human pathogens as well as plant pathogens. Subsequently, we mined into their genomes for discovery of antimicrobial biosynthetic gene clusters (BGCs), that will be further characterized.

Results: The antimicrobial activity of 69 newly isolated strains from a healthy tomato and lettuce phyllosphere against several plant and human pathogens was screened. Three strains with the highest antimicrobial activity were selected and characterized (Bacillus subtilis STRP31, Bacillus velezensis SPL51, and Paenibacillus sp. PL91). All three strains showed a plant growth promotion effect on tomato and lettuce. In addition, genome mining of the selected isolates showed the presence of a large variety of biosynthetic gene clusters. A total of 35 BGCs were identified, of which several are already known, but also some putative novel ones were identified. Further analysis revealed that among the novel BGCs, one previously unidentified NRPS and two bacteriocins are encoded, the gene clusters of which were analyzed in more depth.
\end{abstract}

Conclusions: Three recently isolated strains of the Bacillus genus were identified that have high antagonistic activity against lettuce and tomato plant pathogens. Known and unknown antimicrobial BGCs were identified in these antagonistic bacterial isolates, indicating their potential to be used as biocontrol agents. Our study serves as a strong incentive for subsequent purification and characterization of novel antimicrobial compounds that are important for biocontrol.

Keywords: Biocontrol, Phyllosphere, Bacillus, Paenibacillus, Antimicrobials, biosynthetic gene clusters, NRPs, bacteriocins, RiPPs

*Correspondence: o.p.kuipers@rug.nl

Department of Molecular Genetics, University of Groningen, Groningen,

The Netherlands

\section{Background}

There is an increasing demand by consumers for nutritious foods that improve physical performance and reduce risks of disease. Vegetables represent a widely 
consumed food worldwide. Solanum lycopersicum better known as tomato is one of the most important vegetable plants in the world [1]. Tomato is a healthy food that supplies a wide range of vitamins needed for the organism, since it contains high levels of zinc, potassium, anthocyanins and lycopene, which provide a high antioxidant power. Along with other compounds they reduce risk of contracting cancer, among other benefits according to several epidemiological-food- and health studies $[1,2]$. On the other hand, Lactuca sativa better known as lettuce is another important crop with a growing interest from people due to its healthy and beneficial properties and richness in antioxidants (e.g., vitamins C, E and carotenoids) [3].

Agriculture in the last century has faced multiple challenges, including the need to produce more food to feed a growing population, adapting to climate change, controlling crop diseases that cause significant losses, and adopting more efficient and sustainable production methods. An increased number of fungi, bacteria and viruses are causing plant diseases, several of which are the reason of major economic losses [4]. For this reason, food security has become one of the main points of attention in human-driven development, and therefore any plant pathogen causing substantial crop yield losses needs to be minimized [5]. Currently, tomato and lettuce production losses due to biotic agents (insect pests, microbe -orvirus caused diseases and weeds) have been controlled mainly by spraying crops with a vast amount of synthetic chemical pesticides. However, the extensive use of them caused adverse side effects which represent a serious threat to living organisms and the environment. In addition, for many plant pathogens, fungicide-resistant populations have made many fungicides ineffective [6].

Some human pathogens, such as Bacillus cereus, attach and form biofilms on lettuce leaf surfaces posing a risk of causing disease in humans upon consumption [7]. Klebsiella pneumoniae and Escherichia coli are other known human pathogens that have been reported as causative agents of foodborne diseases, being found in different sources including raw vegetables [8]. Contamination by pathogenic bacteria of vegetables can occur during the harvesting period, post harvesting, handling, storage, transportation, and processing by customers. To ensure safety level of vegetables and others, research of biocontrol agents against human pathogens is needed as well.

It is now well established that plant-associated microorganisms play essential roles in plant health and development and contribute to the environmental equilibrium [9]. The use of beneficial microorganisms is a promising method to fight against crop diseases and increase yields to ensure sufficient crop production [10]. The structural and functional analysis of microbial genomes and the proteins encoded by genes of important plant-associated microbes, which can be possible biocontrol agents will provide insights into several aspects of these molecular interactions and will be crucial for the development of more efficient control measures [11].

Historically, research investigating the factors associated with plant microbe interactions has focused on the rhizosphere, which comprises the area in the soil around plant roots, but much less is known about the phyllosphere. The phyllosphere represents the aboveground or aerial parts of the plants dominated by the leaves in contrast to the rhizosphere. Environmental factors, including UV radiation, changes in relative humidity, temperature, leaf wetness, pollution, nitrogen fertilization as well as biotic factors, such as leaf age and the presence of other microorganisms are factors that microbes endure in such environments [12].

Gram-positive, aerobic spore forming bacteria, like Bacillus and Paenibacillus spp., have been widely reported to be effective in stimulating plant growth and are well known as producers of a broad array of antimicrobials having between 5 and $8 \%$ of the total genome devoted to the biosynthesis of secondary metabolites [13].

The antimicrobials production of Bacillus and closely related species is highly diverse, depending on their biosynthesis pathways, and chemical nature. Antimicrobial compounds can be classified into bacteriocins (both linear and ribosomally synthesized and postranslationally modified peptides (RiPPs)), non- ribosomally synthesized peptides (NRPs), and Polyketides [14]. NRPs and PKs natural products are synthesized via multi domain mega enzymes known as non-ribosomally synthesized peptides synthases (NRPSs) and polyketide synthases (PKSs) which are arranged into units called 'modules' that work in an assembly-line system to build polymeric peptide chains with a determined function [15]. PKSs gather small acetic acid-type acyl construction blocks into polyketides through $\mathrm{C}-\mathrm{C}$ bonds, and NRPSs gather amino acids into peptides through amide bonds. NRPSs and PKSs employ a similar strategy for the biosynthesis of different classes of natural products [16]. On the other hand, bacteriocins are ribosomally synthesized. Bacteriocin BGCs are smaller than those of the previous mentioned compounds, and carry one or more precursor peptide gene(s), which allows for a finer prediction of the end product structure based on the properties of enzymes involved in their biosynthesis and on the chemical structure of the initial peptide substrate. Bacteriocins can be classified in peptides that undergo post-translational modifications (class I) also known as Ribosomally synthesized and Post-translationally modified Peptides 
(RiPPs), or largely unmodified peptides, that sometimes contain disulfide bonds (class II) [17].

Reported and well-known NRPs antimicrobials produced by $B$. subtilis and B. velezensis are cyclic lipopeptides exhibiting strong surfactant and antimicrobial activities, such as surfactins, bacillibactins and fengycins $[14,18]$. B. velezensis, the biocontrol model also employs polyketides such as macrolactin, bacillaene, and difficidin, which play significant roles in both pathogen suppression and plant growth promotion [19]. NRPs like fusaricidin and polymyxin produced by Paenibacillus strains, contributes to antagonism against phyopathogens like Erwinia spp. [20], while well studied Bacillus-originated bacteriocins include subtilosin and subtilomycin [21].

In the last decade volatile compounds (VOCs) produced by some plant-associated bacteria and their biological function, have attracted increased attention. Among other characteristics, they have been proved to have the potential as antimicrobials and have plant growth promoting properties. The VOCs 2,3-butanediol and acetoin, could trigger growth promotion in Arabidopsis thaliana rhizosphere. Bacillus megaterium XTBG34 produces 2-pentylfuran which promotes the growth of Arabidopsis thaliana plants after 15 days of treatment $[22,23]$. In our study we aimed to isolate and screen novel phyllospheric bacteria with antimicrobial properties, and further mined into their genomes to identify known or novel biosynthetic gene clusters (BGCs) that are potentially involved in phytopathogen, and plant-originated human pathogen antagonism.

\section{Results}

Isolation of phyllosperic strains and antimicrobial activity against pathogens

A total of 69 strains were isolated from internal and external leaf tissue from tomato and lettuce plants in early growth stage coming from a small farm in Roden, The Netherlands (Table S1). The antimicrobial activity of the 69 strains was first evaluated against five major tomato and lettuce pathogens, i.e., Rhizoctonia solani [24], Botrytis cinerea [25], and Phytium ultimum [26] and two gram-negative bacterial pathogens, i.e. Pseudomonas syringae pv tomato DC300 [27], and Erwinia corotovora $[28,29]$, covering the two major classes of pathogens, i.e. fungi and Gram-negative bacteria. The three most outstanding strains with the highest antimicrobial activity were selected and their antimicrobial spectrum was extended, to evaluate activity against a wider range of pathogens and to further evaluate properties of plant growth promotion.

In addition, raw and not-processed vegetables, such as tomato and lettuce in salads, are considered as a source of food-borne diseases as well. Some human pathogens can inhabit plants as secondary hosts. In the present study some human pathogens (Klebsiella pneumoniae [30], Bacillus cereus [31] Escherichia coli [32] and Candida albicans [33]) were also included in the antimicrobial activity tests as indicators. Three bacteria with the highest antimicrobial activity were selected: Bacillus subtilis STRP31 isolated from tomato, and Bacillus velezensis SPL51, and Paenibacillus sp. PL91 both isolated from lettuce. These strains can antagonize Gram-positive pathogenic bacteria, Gram-negative pathogenic bacteria, a yeast, and fungal pathogens (Table 1; Fig. 1). The other 66 strains showed a significant lower activity towards bacterial and fungal pathogens tested and had a more limited spectrum of activity.

\section{Genome sequencing of the selected strains and phylogenetic analyses}

The genomes of the selected strains were sequenced, and their DNA sequences were reported previously [34]. Phylogenetic analysis using these whole-genome sequences was conducted with GTDB-Tk v1.5.0 [35] and a phylogenetic dendrogram was constructed with the obtained sequences by IQ-TREE and visualized by iTOL webbased tool [36] (Fig. 2). The species names of the three strains were designated as their most closely related strains namely B. velezensis SPL51, B. subtilis STRP31, and unidentified Paenibacillus sp. PL91.

\section{Plant growth promotion effect}

In vitro experiments were conducted to investigate the effect of volatile organic compounds (VOCs) produced by the three strains (SPL51, STRP31 and PL91) on plant growth of tomato and lettuce plants. Comparisons were made using B. sutbilis 168 as negative control. We observed that the bacterial isolates exerted a significant influence on the increase of plant biomass of tomato and lettuce plants. The plant growth promoting potential of VOCs produced by SPL51, STRP31 and PL91 is depicted in Fig. 3.

\section{Genome mining for BGCs}

A total of 39 gene clusters were found within the three selected strains (Fig. 4A). Among the three strains NRPSs were most abundant, followed by PKSs. The majority of the BGCs found in B. subtilis STRP31 and B. velezensis SPL51 are already known (Fig. 4B). The known BGCs encoded in the genomes are: anabaenopeptin [37], bacilysin [38], bacillibactin [39], difficidin [40], fengycin [41], bacillaene [42], macrolactin $H$ [43], plantazolicin [44], surfactin [45], subtilomycin [46], subtilosin A [47], paeninodin [48] (additional file S2). The highest number of the unknown BCGs belong to strain Paenibacillus sp. 
Table 1 Antimicrobial activity of phyllospheric bacterial strains Bacillus velezensis SPL51, Bacillus subtilis STRP31 and Paenibacillus sp. PL91

\begin{tabular}{|c|c|c|c|c|}
\hline Pathogen types & Species or strains & SPL51 & STRP31 & PL91 \\
\hline \multirow[t]{8}{*}{ Fungi } & Rhizoctonia solani AG2-2 IIIB & ○ & ○ & - \\
\hline & Botrytis cinerea BO5.10 & ○ & ○ & 0 \\
\hline & Verticillium dahlia JR2 & 0 & 0 & O \\
\hline & Fusarium culmorum PV & 0 & - & $\bullet$ \\
\hline & Fusarium oxysporum & 0 & - & $\bullet$ \\
\hline & Alternaria solani & 0 & - & $\bullet$ \\
\hline & Septoria lycopersici & $\bullet$ & O & O \\
\hline & Aspergilus niger & 0 & $\bullet$ & 0 \\
\hline Oomycete & Phytium ultimum P17 & 0 & 0 & $\bullet$ \\
\hline Yeast & Candida albicans & ++ & + & - \\
\hline \multirow[t]{4}{*}{ Gram-negative bacteria } & Escherichia coli WA321 * & + & + & - \\
\hline & Klebsiella pneumoniae * & ++ & + & - \\
\hline & Pseudomonas syringae pv. tomato DC300 & ++ & + & ++ \\
\hline & Erwinia corotovora subsp. brasiliensis LMG21371 & ++ & - & - \\
\hline Gram-positive bacteria & Bacillus cereus ATCC14579* & ++ & + & ++ \\
\hline
\end{tabular}

In the antibacterial assay, no inhibition (-), inhibitory zone $<5 \mathrm{~mm}(+)$, inhibitory zone $\geq 5 \mathrm{~mm}(++)$. In the antifungal assay, no inhibition (O), inhibition $(\bullet)$. * Human pathogens

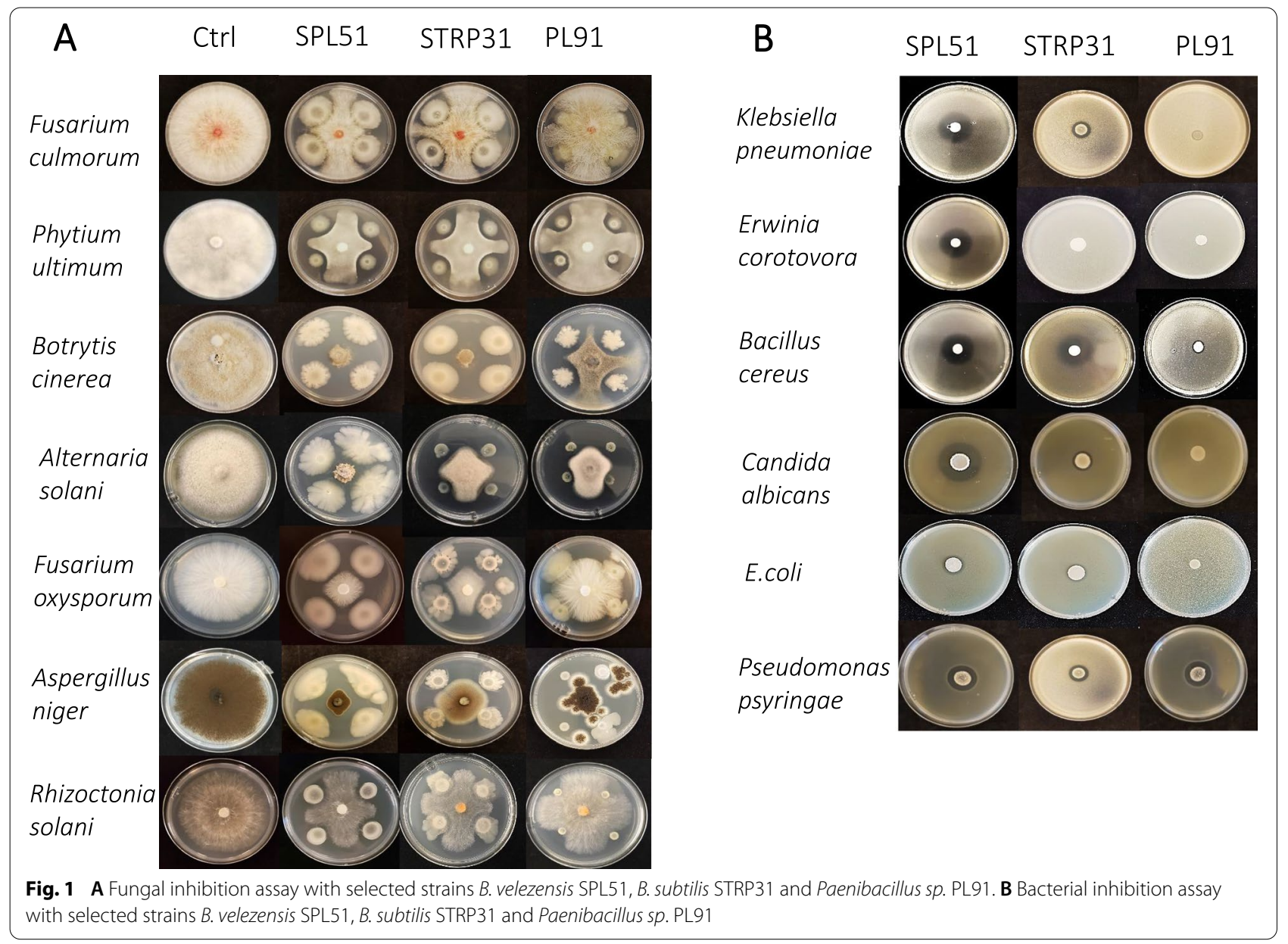




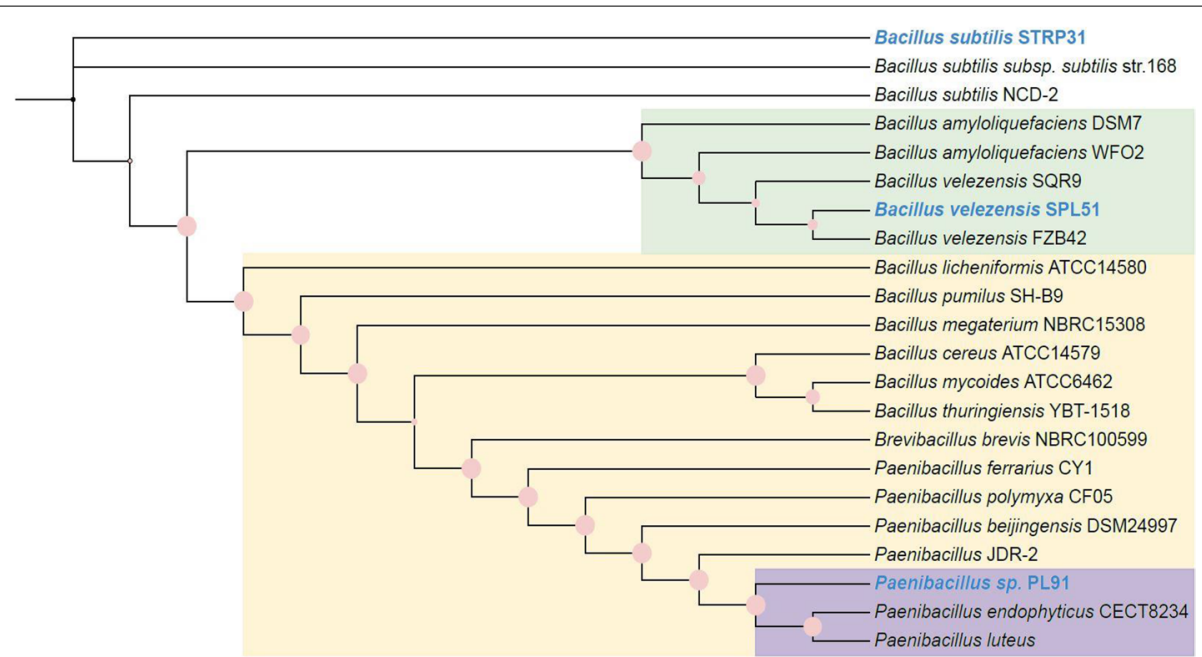

Fig. 2 Phylogenetic analysis of the selected isolated strains with antimicrobial activity in this study. The sequences of reference strains were retrieved from the NCBI database (Table S3 for accession number). The reference strains are in black, and our sequenced strains are highlighted with blue. The pink dots on branches represent bootstrapping values, ranging from 84 to 100
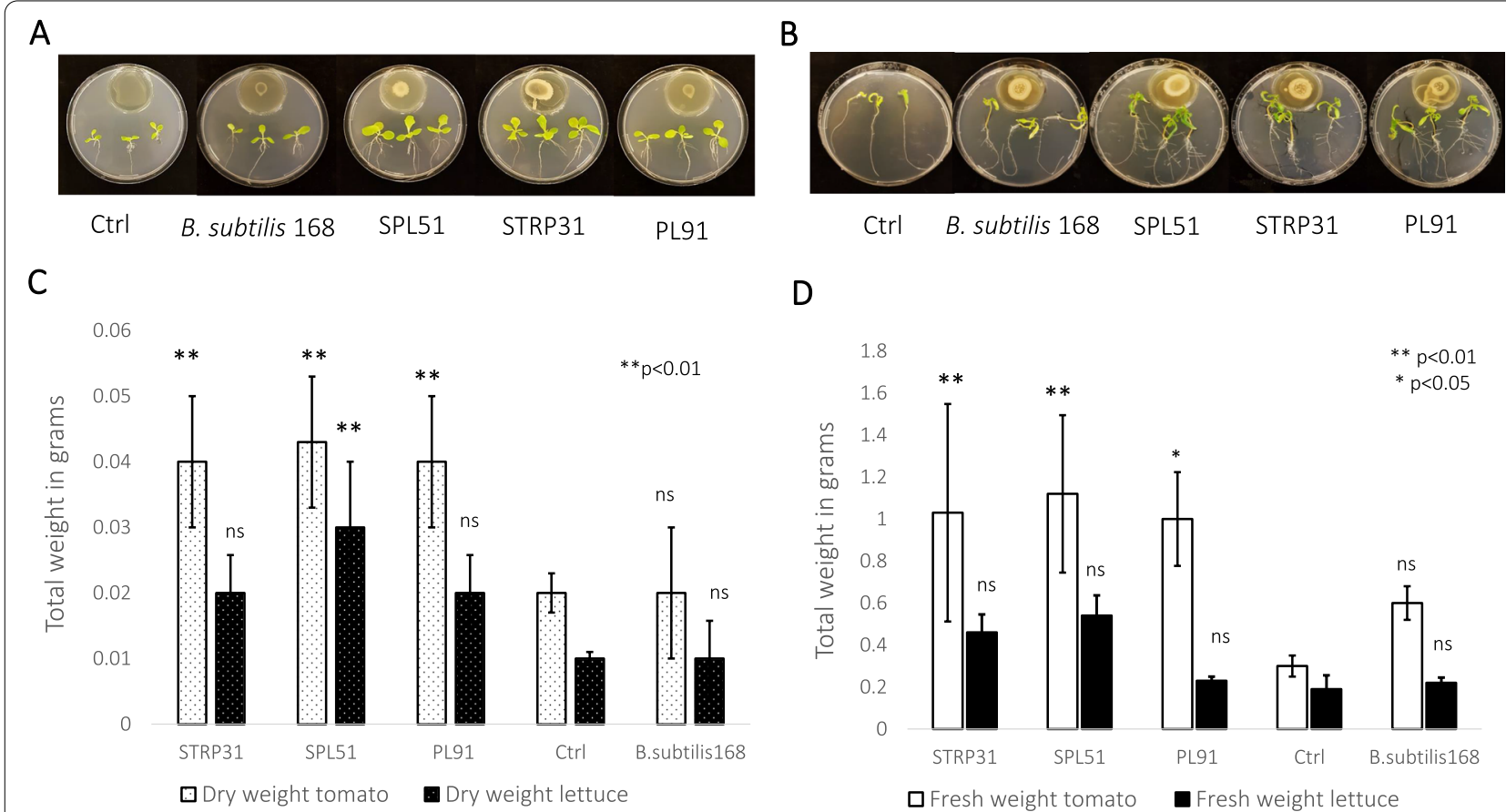

Ctrl B. subtilis $168 \quad$ SPL51 STRP31

PL91

D

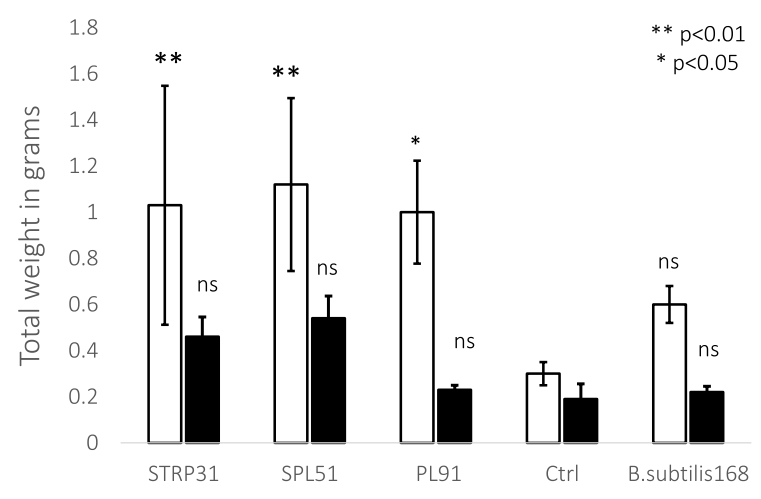

Fig. 3 Plant growth promotion by volatiles. A Phenotypic changes in lettuce seedlings exposed to volatiles emitted by SPL51, STP31, and PL91 strains inoculated on agar $\mathrm{LB}$ medium $(10 \mu \mathrm{OOD}=1.0)$, the control $\mathrm{LB}$ agar only, and B. subtilis 168 used as second control. Pictures were taken 7 days after exposure. B Phenotypic changes in tomato seedlings exposed to volatiles emitted by SPL51, STP31, PL81, and PL91 strains inoculated on agar LB medium ( $10 \mu \mathrm{lOD}=1.0)$ the control LB agar only, and B. subtilis 168 used as second control. Pictures were taken 10 days after exposure. C Biomass dry weight (mean \pm standard error $[\mathrm{SE}], n=4$ ). D Biomass, fresh weight (mean \pm standard error $[\mathrm{SE}], n=4)$. (ns= non-significant, $* * *$ significant, both compared to $\mathrm{Ctrl}$ ) 


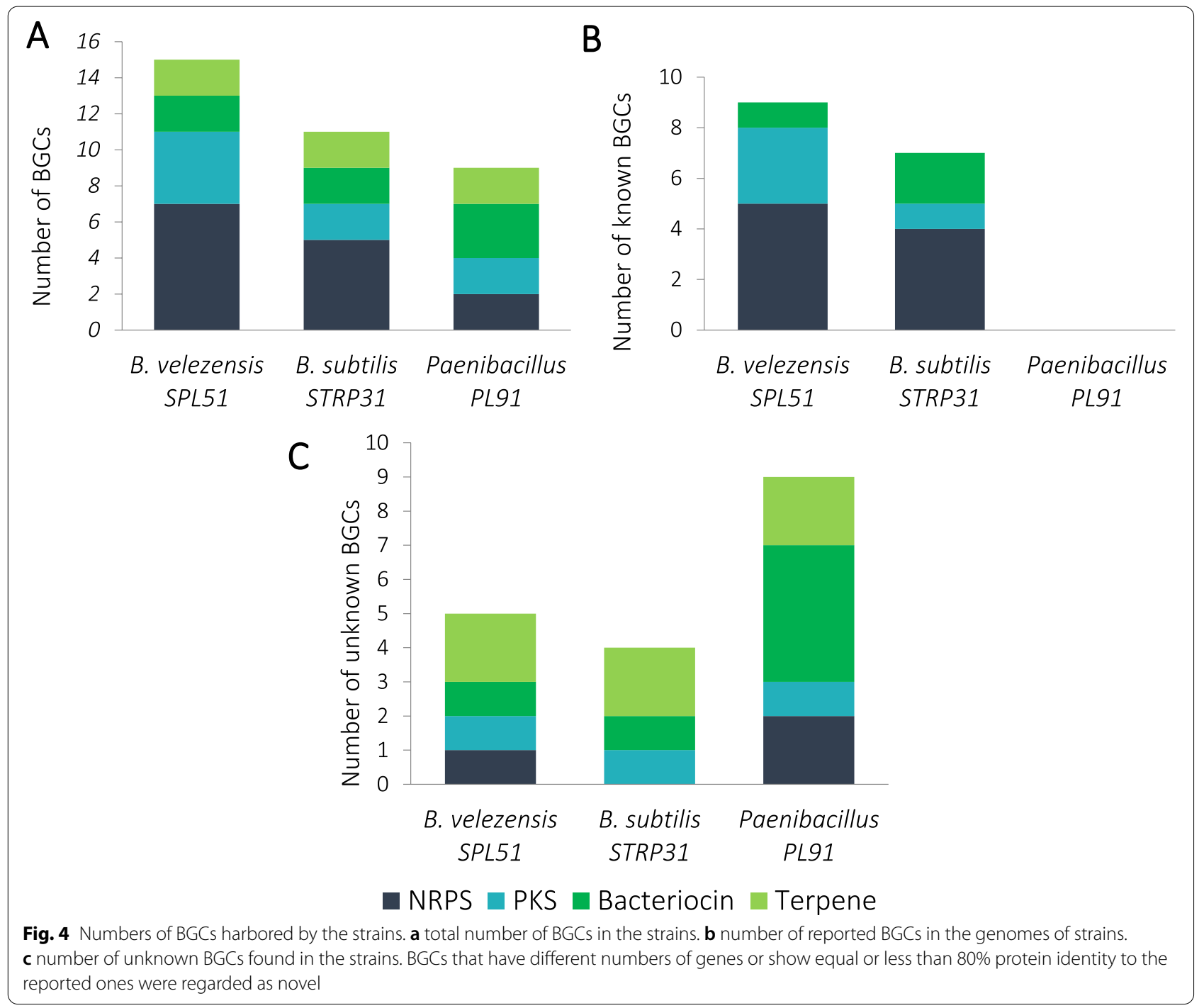

PL91 having nine unknown BGCs, among them are: two NRPSs, one PKs, four bacteriocins and two terpenes. (Fig. 4C).

\section{Putative novel BGCs}

After genome mining, the three more interesting, potentially novel BGCs were chosen based on sequence similarity to other compounds and after comparing the precursor peptides. Selected BGCs are listed in Fig. 5. In the genome of Paenibacillus sp. PL91 a modular NRPS gene cluster with all essential modules (starting module, elongation module, termination module) was found (Fig. 5a). The BGC consists of nine genes and has a total size of $88 \mathrm{~kb}$, it shows $25 \%$ of sequence similarity to the cyclic antimicrobial decapeptide tyrocidine, produced by Brevibacillus brevis NBRC, which exhibits antifungal and antibacterial activity against Gram-positive bacteria.
Tyrocidine A, B and C produced by Brevibacillus spp. share structural similarities, summarized as Cyclo (Phe Pro2 - Phe/Trp3 - Phe/Trp4 - Asn5 - Gln6 - Tyr7 - Val8 - Orn9 - Leu10). Residues 1,2, and 5 to 10 are conserved among the three tyrocidine members produced by Brevibacillus [49]. However, in the NRPs BGC found in PL91 neither the order of genes nor the predicted amino acid composition shows similarity to tyrocidine, indicating the likely novelty of the final product.

Another BGC found in Paenibacillus sp. PL91 was a linear azol(in)e-containing peptide (LAP), an important subgroup of RiPPs with a distinguishing heterocyclic ring of oxazoles and thiazoles derived from serine/ threonine and cysteine by enzymatic cyclodehydration and dehydrogenation [50]. This BGC consists of five genes and has a total size of $41 \mathrm{~kb}$. The precursor peptide encoded by the core biosynthetic gene has 83 amino acids and shows 


\section{a Paenibacillus PL91 (cluster 1) NRPs BGC 88,553 bp

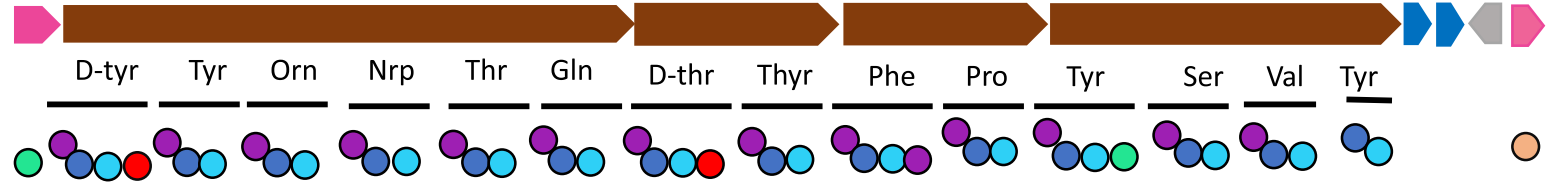 \\ Aminotransferase domain \\ Epimerization domain \\ Peptidyl-carrier protein \\ Adenylation domain

b Paenibacillus PL91 (cluster 5) LAP BGC 41,866 bp

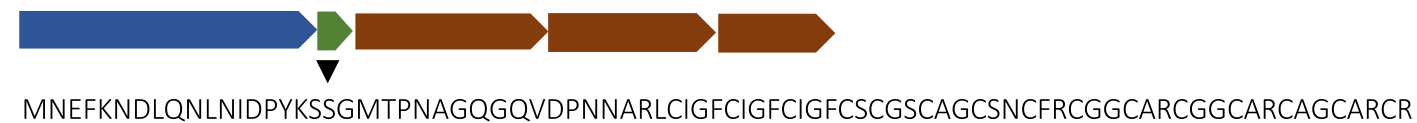

C Paenibacillus PL91 (cluster 9) Lasso peptide BGC 23,910 bp

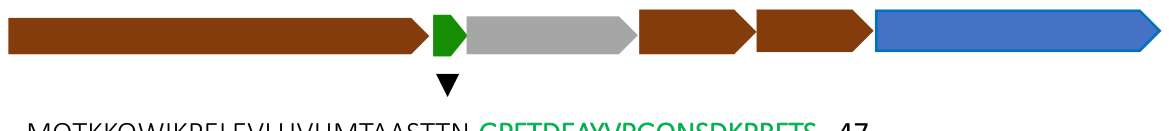

MQTKKQWIKPELEVLHVHMTAASTTN-GPFTDEAYVPGQNSDKPRFTS 47

Paenibacillus dendritiformis C454 Paeninodin

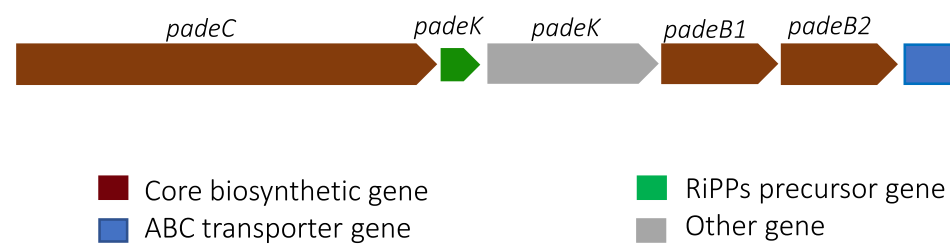

Fig. 5 Potential novel BCGs found in the genomes of the selected strains. a an NRPS present in Paenibacillus PL91. $\mathbf{b}$ a LAP present in Paenibacillus PL91, c A lasso peptide harbored by Paenibacillus PL91 compared to a Paeninodin lasso peptide produced by Paenibacillus dendritiformis C454. The arrows represent the coding regions of the genes identified in the genomes

no similarity with sequences of other bacteriocins. Since it is a bacteriocin it might be possible that it is partially responsible for the antimicrobial activity against Grampositive bacteria, which needs to be further investigated.

An interesting lasso peptide BGC, discovered in Paenibacillus sp. PL91, has $80 \%$ sequence similarity to paeninodin. Paeninodin was firstly discovered in Paenibacillus dendritiformis C454. It was firstly reported in the year 2016 for being a novel lasso peptide tailored by a new class of kinases [48]. Lasso peptide biosynthesis requires at least three genes, referred to as the $\mathrm{A}, \mathrm{B}$, and $\mathrm{C}$ proteins. The $A$ gene encodes the precursor peptide, which is modified by the $B$ and $C$ proteins into the mature natural product [51]. The Lasso peptide BGC found in strain PL91 consists of six genes and has a total size of $23 \mathrm{~kb}$. When we compared lasso peptide BGC in PL91 with the paeninodin lasso peptide, we found they have a highly similar gene organization, and the same number genes too. However, the suggested functionality of the genes is not completely the same. After further analysis with Blastp, the results reveal that the first gene might be an isopeptide-bond forming cyclase protein with cyclization function. The second gene corresponds to the precursor gene. The corresponding precursor peptide compared to the paeninodin produced by Paenibacillus dendritiformis C454 is not the same, thus, this finding suggests a probable novelty of the final product (Fig. 5c). The third gene is suggested to correspond to an aldolase, which is also present in other Paenibacillus spp., while the third gene of Paenibacillus dendritiformis is Pade-K, a gene encoding a kinase, which is not present in the lasso peptide BGC of Paenibacillus sp. PL91. The fourth gene corresponds to be a PqqD family chaperone with a possible function as a maturation enzyme. The fifth gene appears to be a 
Table 2 Proposed functions of ORFs in the putative lasso peptide biosynthetic gene cluster identified by genome mining in Paenibacillus sp. PL91 and reported Bacillus Dendrifitormis C454

\begin{tabular}{llll}
\hline Gene & Paeninodin Paenibacillus dendritiformis C454 & Gene & Lasso peptide BGC Paenibacillus PL91 \\
\hline pade C & Maduration enzyme, cyclization, adenylation & 1 & Isopeptide bond cyclase \\
padeA & Precursor peptide & 2 & Precursor peptide \\
padeK & Putative kinase & 3 & Aldolase \\
padeB1 & Maturation enzyme PqqD superfamily B1 part of "split B", protease & 4 & Maturation enzyme PqqD chaperone \\
padeB2 & Maturation enzyme, transglutaminase like superfamily B2 part of "split B", & 5 & B2 protein maturation enzyme \\
padeD & protease & 6 & ABC transporter ATP-binding protein \\
\hline
\end{tabular}

B2 protein, with a proposed function as a maturation enzyme as well, B2 part of 'split B', protease. Finally, the sixth gene corresponds to be an $\mathrm{ABC}$ transporter, with a proposed export function (Table 2).

\section{Discussion}

In this study we have identified three promising new biocontrol strains, isolated from tomato and lettuce leaves. In addition to their antagonistic activity against several plant and human pathogens, they exert a plant growth promotion effect in tomato and lettuce by the production of VOCs. In a previous study a genome-based analysis from rhizosphere bacteria revealed the presence of several biosynthetic gene clusters (BGCs) for secondary metabolite production which further helped to the pave the road for antimicrobial discovery [52]. In the present study we choose to study a different region, the phyllosphere, which has historically lagged behind our knowledge of the microbiology of the rhizosphere. Insights into the microbial phyllosphere populations and their antimicrobial potential will help us to have a better understanding of the phyllosphere microbiota and develop applications in the promotion of plant growth and plant protection.

We show the presence of a large variety and number of biosynthetic gene clusters among the three selected strains, which were quite different from the best strains found in the rhizosphere [52]. However, various BGCs identified in Bacillus velezensis SPL51 and Bacillus subtilis STRP31 were already known (so called rediscovery), and both strains represent two well studied species with high potential as biocontrol agents to be applied in agriculture, although the ones we isolated could have additional advantages. Paenibacillus sp. PL91 harbored most of the unknown BGCs, including a potentially novel NRPs, and two bacteriocin encoding BGCs (one LAP and one lasso peptide). We suspect that the novel BGCs found in this study have antibacterial and (or) antifungal activity from the antimicrobial assay realized, but this need to be confirmed by future experiments.
Bacteriocins have many properties including their potency (as determined in vitro and in vivo), their low toxicity, the availability of both broad- and narrow-spectrum peptides, the possibility of in situ production by probiotics and the fact that they can be bioengineered. One of the novel BGCs found is a lasso peptide BGC. Lasso peptides are characterized by having peptide bonded proteinogenic amino acids with an extra linkage between the $\mathrm{N}$ - terminus of the peptide and an aspartate or glutamate sidechain, which give them the resembling characteristic topology of a slipknot. Most lasso peptides carry Gly, Cys, Ser or Ala at the N-terminus, and their structure confers them an excellent stability against thermal, chemical, and proteolytic degradation. These features make them attractive for the generation of more potent compounds by engineering. Some lasso peptides are known for their antimicrobial activity, enzyme inhibition, anticancer properties, and antiviral activity. Microcin J25, specialicin, achromosin, sphaericin, actinokineosin, lassomycin are some examples of lasso peptides with antimicrobial activity [53, 54]. This makes them attractive not only for biocontrol purposes but also for pharmaceutical research. The lasso peptide present in strain PL91 has significant differences when compared to other known lasso peptides, suggesting that it is a novel variant of paeninodin, thus making it highly interesting for antimicrobial activity and drug discovery.

On the other hand, the LAP BGC found in PL91 has no similarity with any other known compound. LAPs main components include a precursor peptide (A), and a heterotrimeric enzyme complex composed of a dehydrogenase (B) and cyclodehydratase (C and D). The ribosomal synthesis of the precursor peptide is the first step in the biosynthesis. After the generation of the enzyme-substrate complex, ATP-dependent cyclodehydration takes place, developing into an azoline heterocycle. The most characteristic feature in a LAP is the genetically and biochemically conserved installation of azol(in)e rings on non-macrocyclized natural products), however, other post-translational modifications can be found, such as 
acetylation, methylation, and dehydration [50]. Known LAP include streptolysin S (Streptococcus pyogenes), microcin B17 (Escherichia coli), plantazolicin (Bacillus velezensis), goadsporin (Streptomyces sp. TP- A0584) and clostridiolysin S (Clostridium botulinum), owing to the difficulty inherent to the structure elucidation of heterocycles many LAPs remain unidentified $[53,54]$.

It remains difficult to predict the exact compound products from genome sequence data only. The functionality of the novel BGCs found in our Paenibacillus sp. PL91 strain remains elusive, and further studies will be necessary, including purification of the compound, High Performance Liquid Chromatography (HPLC) mass spectrometry (MS/MS) and Nuclear Magnetic Resonance (NMR) spectroscopy, as well as antimicrobial target specificity of each individual compound. These studies have already been initiated.

\section{Conclusions}

Biological control represents an alternative safe method to counter the extensive use of chemical pesticides which has caused severe environmental and health problems.

In this study, we identified three novel potential biocontrol strains, out of 69 strains screened, that can antagonize phytopathogens and human pathogens, showing potential to be used as biocontrol agents and promote plant growth as well. Further mining into the genomes of the potential biocontrol strains the in-silico prediction revealed a great number of BGCs, including both known and potential novel ones. We found that BGCs in Bacillus species frequently encode conserved known compounds. The Paenibacillus sp. PL91 strain showed the largest number of possible novel BCGs; in our study we found one intact NRPs and two promising bacteriocins. Continued efforts will be directed to purify and characterize these interesting secondary metabolites as well as their determining their contribution to developing biocontrol agents.

\section{Methods}

\section{Collection of plant material and bacterial isolation}

Plant material used in this study came from a small farm in the town of Roden, the Netherlands. Briefly, $2 \mathrm{~g}$ of leaves from tomato and lettuce were macerated to a homogenous liquid state using $5 \mathrm{ml}$ of $10 \mathrm{mM} \mathrm{MgSO} 4$ buffer. The suspension was diluted $10^{3}-10^{6}$ times with $10 \mathrm{mM} \mathrm{MgSO} 4$ buffer, and each dilution was exposed to heat treatment of $80{ }^{\circ} \mathrm{C}$ for $15 \mathrm{~min}$ and subsequently spread on LB agar plates and incubated at $28{ }^{\circ} \mathrm{C}$ for the next $72 \mathrm{~h}$. Colony growth was monitored and each colony was isolated and cultured on a separate plate. Stocks were created by using glycerol at $80 \%$ solutions and stored at $-80{ }^{\circ} \mathrm{C}[34]$.

\section{Phylogenetic analysis}

The genome sequences of the selected strains used in our study were determined as described previously [34]. Genome-scale comparison of the bacterial strains and other relevant strains were conducted GTDB-Tk v1.5.0 [36]. A multiple sequence alignment of the 118 identified markers from 22 genomes was generated. It was employed to build a maximum likelihoodphylogenetic tree by using IQ-TREE multicore version 1.6.12 [55]. Substitution models LG+F+R4 was selected by ModelFinder [56]. iTOL web-based tool [36] was used for tree visualization, using the previous alignment.

\section{Antimicrobial activity assays}

Fungal inhibition assay

Each fungus was inoculated in a PDA agar plate and incubated at $28{ }^{\circ} \mathrm{C}$ for specific time according to the fungus growth rate. Rhizoctonia solani, Phytium ultimum, Aspergillus Niger and Fusarium culmorum 3 days of incubation, and Botrytis cinerea, Alternaria Solani and Fusarium oxysporum 5 days of incubation. Briefly, an agar plug (5 $\mathrm{mm}$ diameter) with fungal hyphae was inoculated into a PDA plate at the center, and $5 \mu \mathrm{l}$ of $1 \times 10^{8}$ cells $/ \mathrm{ml}$ overnight culture of each isolated strain of the collection isolates, were subsequently spotted $2 \mathrm{~cm}$ away from the plug in 4 corners symmetrically. Plates were sealed with parafilm and incubated at $28{ }^{\circ} \mathrm{C}$ for each fungus specific growth time, and inhibition was documented.

Verticillum dahliae and Septoria licopersici fungal inhibition assay

Slow growing fungi Verticullium dahliae and Septoria licopersici were incubated for one month at $28{ }^{\circ} \mathrm{C}$. Plugs were cutted from 3 whole plates with each different fungus, and then vortexed in $10 \mathrm{ml}$ of sterile water to collect the spores, mixed with $15 \mathrm{ml}$ PDA agar, and the solution poured into plates. After drying $5 \mu \mathrm{l}$ of $1 \times 10^{8}$ cells $/ \mathrm{ml}$ overnight culture of SPL51, STRP31 andPL91 strain was spotted at the center, followed by two to eight days incubation at $28{ }^{\circ} \mathrm{C}$, and inhibition was registered.

\section{Bacterial inhibition assay}

To test antibacterial activity, overnight cultures of bacterial pathogens were grown until $\mathrm{OD}_{600}=1.0$; then mixed with LB agar media, at a final concentration of $1 \times 10^{6}$ cells $/ \mathrm{ml}$. The mixed media was poured into petri dishes to get pathogen- fusion agar plates. Subsequently $7 \mu \mathrm{l}$ of $\left(1 \times 10^{8}\right.$ cells $\left./ \mathrm{mL}\right)$ culture of each isolated strain was inoculated at the center of the plate, followed by incubation between 24 and $48 \mathrm{~h}$. The presence or absence of a halo was monitored, and the inhibition zone was determined in $(\mathrm{mm})$. 


\section{Plant growth promoting assay by volatile compounds (VOCs) in vivo}

Tomato seeds were sterilized by submerging them in $2 \%$ sodium hypochlorite for $15 \mathrm{~min}$ and then washed with sterile water 5 times to remove completely sodium hypochlorite. Seeds were germinated on large Petri dishes with Murashigue Skoog medium (Duchefa Biochemie) and incubated in a climate chamber with the next conditions: Light-time $(16 \mathrm{~h})$ at $24+/-2$ Celsius degrees, 4000-6000 lx (or photon flux density of 200 umol m-2 S-1); Dark time (8 h) at $21+/-2$ Celsius degrees; humility $75+/-5 \%$. Once seeds germinated after 3 or 6 days (lettuce and tomato respectively), seedlings were transferred to a new MS Petri dish and $10 \mu \mathrm{l}$ of overnight cultures with $\mathrm{OD}_{600} 1.0$ were inoculated onto a separate small petridish inside the MS plate creating a separate compartment in order to evaluate plant growth promotion by volatile compounds. Plates were sealed with parafilm and after co-culture of 7 and 10 days (lettuce and tomato respectively) in the same conditions mentioned before, fresh weight and dry weight of the plants was measured. All methods were performed in accordance with the relevant guidelines/regulations/legislation. A one-way ANOVA analysis using a post-hoc Tukey HSD test was conducted $(P<0.05)$ to evaluate the significance.

\section{Genome mining for BGCs}

The genome mining of biosynthetic gene clusters of antimicrobial compounds was conducted with antiSMASH 5.0 [57] and BAGEL4 [58]. Before applying to the pipelines, the draft genome of each strain which was originally assembled with Spades [35] was re-assembled into a pseudomolecule using closely related strains as a reference using Medusa web server. When a draft genome is created, individual reads of DNA are second assembled into contigs, which, have gaps between them. Medusa web server bridge the gaps between these contigs to create a scaffold, is a draft genome scaffolder that uses multiple reference genomes in a graphbased approach to determine the correct order and orientation of the contigs [59] (http://combo.dbe.unifi. it/medusa). The genes predicted from both pipelines were further confirmed with protein BLAST. BGCs that showed equal or less than $80 \%$ protein identity to the reported ones were regarded as novel.

\section{Abbreviations}

BCAs: Biocontrol agents; BGCs: Biosynthetic gene clusters; NRPs: Nonribosomal peptides; NRPSs: Nonribosomal peptide synthetases; PKs: Polyketides; PKSs: Polyketide synthetases; RiPPs: Ribosomally produced and posttranslationally modified peptides; VOCs: Volatile organic compounds; A: Adenylation; ACP: Acyl-carrier protein; AT: Acyltransferase; Atd: Trans-acyltransferase docking; C: Condensation; CAL: Co-enzyme A ligase domain; DH: Dehydratase; E: Epimerization; ISR: Induced systemic resistance; KR: Keto-reductase; KS: Keto-synthase; PCP: Peptidyl carrier protein; PGPR: Plant growth-promoting rhizobacteria; TE: Thioesterase.

\section{Supplementary Information}

The online version contains supplementary material available at https://doi. org/10.1186/s12864-022-08392-0.

Additional file 1.

\section{Acknowledgements}

We are grateful to Prof. Jos Raaijmakers of NIOO-KNAW, Prof. Gilles van Wezel of Leiden University, and Prof. Nicholas J Talbot of the University of Exeter for the kind gifts of plant pathogens. We kindly appreciate Dr. Sjoerd van der Meulen for providing us the plants from his farm in Roden, The Netherlands.

\section{Authors' contributions}

Conceived and designed the project: CM, LZ and OK. Supervised the project: OK. Conceived and designed the experiments: CM, LZ and OK. Execution of experimental work: CM, LZ, YY and interpretation of the data: $C M, L Z, Y Y$ and OK. Manuscript writing: CM. Corrected the manuscript: CM, LZ, OK. All authors read and approved the final manuscript.

\section{Funding}

Claudia Y. Muñoz was financially supported by the Mexican National Council for Science and Technology (CONACYT) scholarship (471987).

Lu Zhou was financially supported by the China Scholarship Council (201606910037).

Yunhai Yi was financially supported by the China Scholarship Council (201904910477)

\section{Availability of data and materials}

The whole genome data are available at DDBJ/EMBL/GenBank under the bio project accession PRJNA623850 ( https://www.ncbi.nlm.nih.gov/bioproject/ 623850 ).

\section{Declarations}

Ethics approval and consent to participate

All methods were performed in accordance with the relevant guidelines/ regulations/legislation.

\section{Consent for publication}

Not applicable.

\section{Competing interests}

The authors declare that they have no conflict interests.

Received: 6 December 2021 Accepted: 9 February 2022

Published online: 21 February 2022

\section{References}

1. Kimura S, Sinha N. Tomato (Solanum lycopersicum): A Model Fruit-Bearing Crop. CSH Protoc. 2008;2008:pdb.emo 105.

2. Campbell JK, Canene-Adams K, Lindshield BL, Boileau TW, Clinton SK, Erdman JW Jr. Tomato phytochemicals and prostate cancer risk. J Nutr. 2004;134(12 Suppl):3486S-92S.

3. Nicolle C, Cardinault N, Gueux E, Jaffrelo L, Rock E, Mazur A, Amouroux $P$, Rémésy $C$. Health effect ofvegetable-based diet: lettuce consumption improves cholesterol metabolism andantioxidant status in the rat. Clin Nutr. 2004:23(4):605-14. 
4. Nazarov PA, Baleev DN, Ivanova MI, Sokolova LM, Karakozova MV. Infectious Plant Diseases: Etiology, Current Status, Problems and Prospects in Plant Protection. Acta Naturae. 2020;12(3):46-59.

5. Smith P, Gregory PJ. Climate change and sustainable food production. Proc Nutr Soc. 2013;72(1):21-8.

6. Chen X, Pizzatti C, Bonaldi M, Saracchi M, Erlacher A, Kunova A, Berg G, Cortesi P. Biological Control of Lettuce Drop and Host Plant Colonization by Rhizospheric and Endophytic Streptomycetes. Front Microbiol. 2016;7:714.

7. Elhariry HM. Attachment strength and biofilm forming ability of Bacillus cereus on green-leafy vegetables: cabbage and lettuce. Food Microbiol. 2011;28(7):1266-74.

8. Puspanadan S, Afsah-Hejri L, Loo Y, Nillian E, Kuan C, Goh SG, Chang WS, Lye YL, John YT, Rukayadi Y, Yoshitsugu N, Nishibuchi M, Son R. Detection of Klebsiella pneumoniae in raw vegetables using Most Probable NumberPolymerase Chain Reaction (MPN-PCR). Int Food Res J. 2012;19:1757-62.

9. Ongena M, Jacques P. Bacillus lipopeptides: versatile weapons for plant disease biocontrol. Trends Microbiol. 2008;16(3):115-125.

10. Heydari A, Pessarakli M. A Review on Biological Control of Fungal Plant Pathogens Using Microbial Antagonists. J Biol Sci J Biol Sci Vol. 2010;10(4):273-90.

11. Montesinos E. Plant-associated microorganisms: a view from the scope of microbiology. International Microbiology. 2003; 6(4), 221-223.

12. Vorholt JA. Microbial life in the phyllosphere. Nat Rev Microbiol. 2012;10(12):828-40.

13. Fira D, Dimkić I, Berić T, Lozo J, Stanković S. Biological control of plant pathogens by Bacillus species. J Biotechnol. 2018;285:44-55.

14. Zhao X, Kuipers OP. Identification and classification of known and putative antimicrobial compounds produced by a wide variety of Bacillales species. BMC Genomics. 2016;17(1):882.

15. Weissman KJ. The structural biology of biosynthetic megaenzymes. Nat Chem Biol. 2015;11(9):660-670.

16. Miyanaga A, Kudo F, Eguchi T. Protein-protein interactions in polyketide synthase-nonribosomal peptide synthetase hybrid assembly lines. Nat Prod Rep. 2018:35(11):1185-209.

17. Montalbán-López M, Scott TA, Ramesh S, et al. New developments in RiPP discovery, enzymology and engineering. Nat Prod Rep. 2021:38(1):130-239.

18. Kaspar F, Neubauer P, Gimpel M. Bioactive Secondary Metabolites from Bacillus subtilis: A Comprehensive Review. J Nat Prod. 2019;82(7):2038-53.

19. Rabbee MF, Ali MS, Choi J, Hwang BS, Jeong SC, Baek KH. Bacillus velezensis: A Valuable Member of Bioactive Molecules within Plant Microbiomes. Molecules. 2019;24(6):1046.

20. Niu B, Vater J, Rueckert C, et al. Polymyxin $P$ is the active principle in suppressing phytopathogenic Erwinia spp. by the biocontrol rhizobacterium Paenibacillus polymyxa M-1. BMC Microbiol. 2013;13:137.

21. Dunlap CA., Schisler DA., Bowman MJ., \& Rooney AP. Genomic analysis of Bacillus subtilis $\mathrm{OH} 131.1$ and co-culturing with Cryptococcus flavescens for control of Fusarium head blight. Plant Gene. 2015; 2, 1-9.

22. Avalos M, van Wezel GP, Raaijmakers JM, Garbeva P. Healthy scents: microbial volatiles as new frontier in antibiotic research?. Curr Opin Microbiol. 2018:45:84-91.

23. Zou C, Li Z, Yu D. Bacillus megaterium strain XTBG34 promotes plant growth by producing 2-pentylfuran. J Microbiol. 2010;48(4):460-6.

24. Ajayi-Oyetunde OO., and Bradley CA. Rhizoctonia solani: taxonomy, population biology and management of rhizoctonia seedling disease of soybean. Plant Pathol. 2018;67: 3-17.

25. Rupp S, Weber RW, Rieger D, Detzel P, Hahn M. Spread of Botrytis cinerea Strains with Multiple Fungicide Resistance in German Horticulture. Front Microbiol. 2017;7:2075.

26. Salman M, Abuamsha R. Potential for integrated biological and chemical control of damping-off disease caused by Pythium ultimum in tomato. BioControl. 2012;57:711-8.

27. Xin XF, He SY. Pseudomonas syringae pv. tomato DC3000: a model pathogen for probing disease susceptibility and hormone signaling in plants. Annu Rev Phytopathol. 2013;51:473-98.

28. Alippi AM, Bó ED, Ronco LB, Casanova PE, Aguilar OM. Tomato as a New Host of Erwinia carotovora subsp. carotovora in Argentina. Plant Dis. 1997:81(2):230.
29. Toth IK, Bell KS, Holeva MC, Birch PR. Soft rot erwiniae: from genes to genomes. Mol Plant Pathol. 2003;4(1):17-30.

30. Hartantyo SHP, Chau ML, Koh TH, et al. Foodborne Klebsiella pneumoniae: Virulence Potential, Antibiotic Resistance, and Risks to Food Safety. J Food Prot. 2020;83(7):1096-103.

31. Tewari A, Abdullah S. Bacillus cereus food poisoning: international and Indian perspective. J Food Sci Technol. 2015;52(5):2500-2511.

32. Dublan Mde L, Ortiz-Marquez JC, Lett L, Curatti L. Plant-adapted Escherichia coli show increased lettuce colonizing ability, resistance to oxidative stress and chemotactic response. PLoS One. 2014;9(10):e110416.

33. Rajkowska K, Kunicka-Styczyńska A. Typing and virulence factors of foodborne Candida spp. isolates. Int J Food Microbiol. 2018;279:57-63.

34. Muñoz CY, de Jong A, Kuipers OP. Draft genome sequences of a Bacillus subtilis strain, a Bacillus velezensis strain, a Paenibacillus strain, and an Acinetobacter baumannii strain, all isolated from the phyllosphere of Lactuca sativa or Solanum lycopersicum. Microbiol Resour Announc. 2021; 10:e01092-20.

35. Chaumeil PA, Mussig AJ, Hugenholtz P, Parks DH. GTDB-Tk: a toolkit to classify genomes with the Genome Taxonomy Database. Bioinformatics. 2019;36(6):1925-7.

36. Letunic I, Bork P. Interactive tree of life (iTOL) v3: an online tool for the display and annotation of phylogenetic and other trees. Nucleic Acids Res. 2016;44(W1):W242-W245.

37. Entfellner E, Frei M, Christiansen G, Deng L, Blom J, Kurmayer R. Evolution of Anabaenopeptin Peptide Structural Variability in the Cyanobacterium Planktothrix. Front Microbiol. 2017:8:219.

38. Özcengiz G, Öğülür İ. Biochemistry, genetics and regulation of bacilysin biosynthesis and its significance more than an antibiotic. N Biotechnol. 2015;32(6):612-619.

39. May JJ, Wendrich TM, Marahiel MA. The dhb operon of Bacillus subtilis encodes the biosynthetic template for the catecholic siderophore 2,3-dihydroxybenzoate-glycine-threonine trimeric ester bacillibactin. J Biol Chem. 2001;276(10):7209-17.

40. Wu L, Wu H, Chen L, Yu X, Borriss R, Gao X. Difficidin and bacilysin from Bacillus amyloliquefaciens FZB42 have antibacterial activity against Xanthomonas oryzae rice pathogens. Sci Rep. 2015;5:12975.

41. Sur S, Romo TD, Grossfield A. Selectivity and Mechanism of Fengycin, an Antimicrobial Lipopeptide, from Molecular Dynamics. J Phys Chem B. 2018;122(8):2219-26.

42. Butcher RA, Schroeder FC, Fischbach MA, et al. The identification of bacillaene, the product of the PksX megacomplex in Bacillus subtilis. Proc Natl Acad Sci U S A. 2007;104(5):1506-9.

43. Yuan J, Zhao M, Li R, et al. Antibacterial Compounds-Macrolactin Alters the Soil Bacterial Community and Abundance of the Gene Encoding PKS. Front Microbiol. 2016;7:1904.

44. Scholz R, Molohon KJ, Nachtigall J, et al. Plantazolicin, a novel microcin B17/streptolysin S-like natural product from Bacillus amyloliquefaciens FZB42. J Bacteriol. 2011;193(1):215-24.

45. Zhi Y, Wu Q, Xu Y. Genome and transcriptome analysis of surfactin biosynthesis in Bacillus amyloliquefaciens MT45. Sci Rep. 2017; 7, 40976.

46. Phelan RW, Barret M, Cotter PD, et al. Subtilomycin: a new lantibiotic from Bacillus subtilis strain MMA7 isolated from the marine sponge Haliclona simulans. Mar Drugs. 2013;11(6):1878-1898.

47. Babasaki K, Takao T, Shimonishi Y, Kurahashi K, Subtilosin A. a new antibiotic peptide produced by Bacillus subtilis 168: isolation, structural analysis, and biogenesis. J Biochem. 1985;98(3):585-603.

48. Zhu S, Hegemann JD, Fage CD, et al. Insights into the Unique Phosphorylation of the Lasso Peptide Paeninodin. J Biol Chem. 2016;291(26):13662-13678

49. Yang $X$, Yousef AE. Antimicrobial peptides produced by Brevibacillus spp.: structure, classification and bioactivity: a mini review. World J Microbiol Biotechnol. 2018;34(4):57.

50. Arnison PG, Bibb MJ, Bierbaum G, et al. Ribosomally synthesized and post-translationally modified peptide natural products: overview and recommendations for a universal nomenclature. Nat Prod Rep. 2013;30(1):108-60.

51. Letzel AC, Pidot SJ, Hertweck C. Genome mining for ribosomally synthesized and post-translationally modified peptides (RiPPs) in anaerobic bacteria. BMC Genomics. 2014;15(1):983. 
52. Zhou L, Song C, Li Z. et al. Antimicrobial activity screening of rhizosphere soil bacteria from tomato and genome-based analysis of their antimicrobial biosynthetic potential. BMC Genomics. 2021;22:29.

53. Cheng C, Hua ZC. Lasso Peptides: Heterologous Production and Potential Medical Application. Front Bioeng Biotechnol. 2020 Sep 28:8:571165.

54. Maksimov MO, Link AJ. Prospecting genomes for lasso peptides. J Ind Microbiol Biotechnol. 2014:41(2):333-44.

55. Nguyen LT, Schmidt HA, von Haeseler A, Minh BQ. IQ-TREE: a fast and effective stochastic algorithm for estimating maximum-likelihood phylogenies. Mol Biol Evol. 2015;32(1):268-74.

56. Kalyaanamoorthy S, Minh BQ, Wong TKF, von Haeseler A, Jermiin LS. ModelFinder: fast model selection for accurate phylogenetic estimates. Nat Methods. 2017;14(6):587-9.

57. Blin K, Shaw S, Steinke K, et al. antiSMASH 5.0: updates to the secondary metabolite genome mining pipeline. Nucleic Acids Res. 2019:47(W1):W81-W87.

58. van Heel AJ, de Jong A, Song C, Viel JH, Kok J, Kuipers OP. BAGEL4: a userfriendly web server to thoroughly mine RiPPs and bacteriocins. Nucleic Acids Res. 2018;46(W1):W278-81.

59. Bosi E, Donati B, Galardini M, et al. MeDuSa: a multi-draftbased scaffolder. Bioinformatics. 2015;31(15):2443-51.

\section{Publisher's Note}

Springer Nature remains neutral with regard to jurisdictional claims in published maps and institutional affiliations.

- fast, convenient online submission

- thorough peer review by experienced researchers in your field

- rapid publication on acceptance

- support for research data, including large and complex data types

- gold Open Access which fosters wider collaboration and increased citations

- maximum visibility for your research: over $100 \mathrm{M}$ website views per year

At BMC, research is always in progress.

Learn more biomedcentral.com/submissions 